\title{
Spatial solitons in chiral nematics
}

\author{
Urszula A. Laudyn, Katarzyna Jaworowicz, Mirosław A. Karpierz \\ Warsaw Univeristy of Technology, Faculty of Physics \\ Koszykowa 75, 00-662 Warsaw - Poland \\ http://www.if.pw.edu.pl/ karpierz
}

\begin{abstract}
In this work we present for the first time experimental results of measurements of spatial solitons (nematicons) in chiral nematic liquid crystalline film. We measured the propagation of light beam at the distance of a few millimeters and the nonlinear self-focusing was observed for a light power of order of a few tenths of milliwats. Additional, in a waveguide induced by the nematicon, other low-power light beam (signal beam) with different wavelength was trapped. There were also observed the changes of the nematicon direction by changing the position across the nematics layer. The experimental results are in a good agreement with theoretical predictions.
\end{abstract}

Keywords: nematicons, reorientational nonlinearity, spatial solitons.

Reorientational nonlinearity in nematic liquid crystals (NLCs) is a source of various phenomena [1], including the creation of spatial solitons, called as nematicons [2,3]. Spatial solitons are light beams which do not spread due to the balance between diffraction and nonlinear self-focusing effects. They can be applied in all-optical switching and routing systems. Special interest in forming of spatial solitons in NLCs is connected with unique properties of nematicons: among others low light power required to their formation, possibility of steering by external fields and polarization sensitivity. Nematicons have already been analyzed in plane waveguides with homeotropic textures [4-5], in layers with planar texture and external electric field [6-8] and in capillaries with axial orientation [9-10]. It has been established that the light beam with a power in the order of a few milliwats can form nematicons at a distance of a few millimeters. The electrically driven arrays of waveguides in NLCs, leading to the discrete solitons have been also demonstrated [11-12]. Nematicons have been also predicted theoretically and observed experimentally in twisted nematic layers, where the direction of NLC orientation is parallel to the surrounding glass plates and twisted respectively in both plates [13-16].

In this paper, for the first time to our best knowledge, we demonstrate experimentally the existence of spatial solitons in chiral nematic liquid crystals (ChNLCs) $[17,18]$. In the analyzed configuration (presented in Fig.1) a light beam propagates in the z-direction parallel to the glass plates. Assuming that the light is polarized along y-direction, means that the beam propagates in the layer close to the plane, where the ChNLCs molecules are parallel to the yaxis. In principle, the light beam propagation and nematicons creation in ChNLCs are similar to those in twisted nematics layer (TNs) [16]. However, the configuration with ChNLCs offers some new opportunities in comparison with TNs. This is connected with the fact, that the width of the guiding layer (in x-direction) is not only determined by the width of the sample $d$ (like in TNs) but also by the chirality pitch. As a result, in ChNLCs it is much easier to choose the proper width of the guiding layer and as a consequence the proper nonlinearity 
strength, than in TNs. It is also possible to utilize multi-layers for propagation of independent or interacting nematicons.

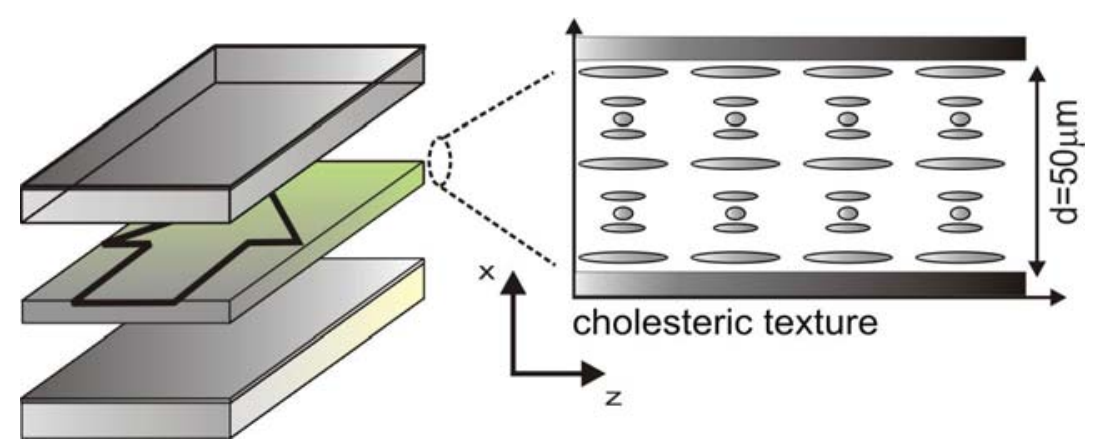

Fig.1 Configuration of the analyzed chiral nematic liquid crystal cell.

The orientation of ChNLC is determined by anchoring conditions at boundaries where the interaction with the cell walls (glass plates) introduces boundary orientation. The orientation angle between molecules direction and y axis is initially equal to: $\theta(x)=(2 \pi m / d) x$, where $m$ is an integer number. Consequently the electric permittivity tensor in such medium has the form:

$$
\varepsilon=\left(\begin{array}{ccc}
\varepsilon_{\perp} & 0 & 0 \\
0 & \varepsilon_{\perp}+\Delta \varepsilon \cos ^{2} \theta & \Delta \varepsilon \sin \theta \cos \theta \\
0 & \Delta \varepsilon \sin \theta \cos \theta & \varepsilon_{\perp}+\Delta \varepsilon \sin ^{2} \theta
\end{array}\right)
$$

where: $\Delta \varepsilon=\varepsilon_{\|}-\varepsilon_{\perp}$ represents an optical anisotropy, $\varepsilon_{\perp}=n_{o}^{2}$ is an ordinary and $\varepsilon_{\|}=n_{e}^{2}$ is an extraordinary electric permittivity. The polarized light beam with dominating $\mathrm{E}_{\mathrm{y}}$ component of the electric field is assumed. It means, that the refractive index varies across the sample from $n_{\mathrm{o}}$ in planes where $\theta=\pi / 2$, to $n_{\mathrm{e}}$ in planes where $\theta=0$. This is equivalent to the planar waveguide with gradient refractive index. Therefore, the light beam is guided in thin layer around $\theta=0$ and it does not diffract in $x z$ plane. However in $y z$ plane the light beam diffracts, as the refractive index distribution is constant. Taking into consideration the light behavior in $y z$ plane, it can be assumed that beam profile in $x$ direction is unchanged during propagation.

In a nonlinear regime, for higher light intensity, the liquid crystal molecules are forced to reorient to be parallel to the electric field. The value of $E_{\mathrm{x}}$ and $E_{\mathrm{z}}$ components are assumed to be much weaker than the $E_{\mathrm{y}}$ component, and the $E_{\mathrm{x}}$ component has a $\pi / 2$ phase shift versus the $E_{\mathrm{y}}$ [13]. This causes that the reorientation will behave only in $y z$ planes and will be connected with the twist deformation. Moreover, the significant changes in orientation angle will be in the directions of $x$ and $y$ axis. In such a case the minimization of the free energy leads to the Euler-Lagrange equation in the form:

$$
\frac{\partial^{2} \theta}{\partial x^{2}}+\frac{\partial^{2} \theta}{\partial y^{2}}+\frac{\varepsilon_{0} \Delta \varepsilon}{4 K_{22}}\left[\left(E_{z}^{2}-E_{y}^{2}\right) \sin 2 \theta+2 E_{z} E_{y} \cos 2 \theta\right]=0,
$$

where $K_{22}$ is a twist deformation elastic constant. Although the above equation is formally the same as for NLCs, its periodic solutions are proper for ChNLCs. With increase of the light intensity, the width of the region with $\theta=0$ increases and it also increases the effective 
refractive index. This results in the self-focusing of the light beam in y-direction and finally creation of the nematicon.

The analyzed sample consisted of two glass plates glued together, with a gap between the plates which was controlled by spacers. The cell was filled through a capillary effect with 6CHBT nematics (4-trans-4'-n-hexyl-cyclohexyl-isothiocyanatobenzene) doped with a chiral material. The width of the cells was approximately $50 \mu \mathrm{m}$ and the pitch of the ChNLC was about $25 \mu \mathrm{m}$ in free space - it corresponds to approximately $(4 \div 5) \pi$ range of the orientation angle $\theta$ in the cell. Measurements were carried out for a light with the TE polarization, i.e. with dominant $E_{\mathrm{y}}$ component, and were compared with results obtained for perpendicular TM polarization.

Light beam propagation was investigated experimentally in the setup schematically presented in the Fig. 2. Light beam from a linearly polarized argon laser $(\lambda=514 \mathrm{~nm})$ was injected into the liquid crystal cell by means of $15 \mathrm{x}$ microscope objective lens. The $15 \mathrm{x}$ objective lens focused light into a spot with a waist of about $2 \mu \mathrm{m}$. This value was estimated by measuring the divergence of the beam after the focal point during linear propagation in liquid crystal film. The position of the cell was adjusted by microtranslation stages in the $x, y$ and $z$ direction and rotational stages (rotation around $x$ and the $y$ axis). An additional rotator was used to control the polarization of light. NLCs scatter light due to the fluctuations in director orientation. Thanks to this the beam traveling laterally in the cell was observed by $20 \mathrm{x}$ microscope objective lens connected with CCD camera mounted in a $(x, y, z)$ stage. Acquisition and analysis was made by a computer system.

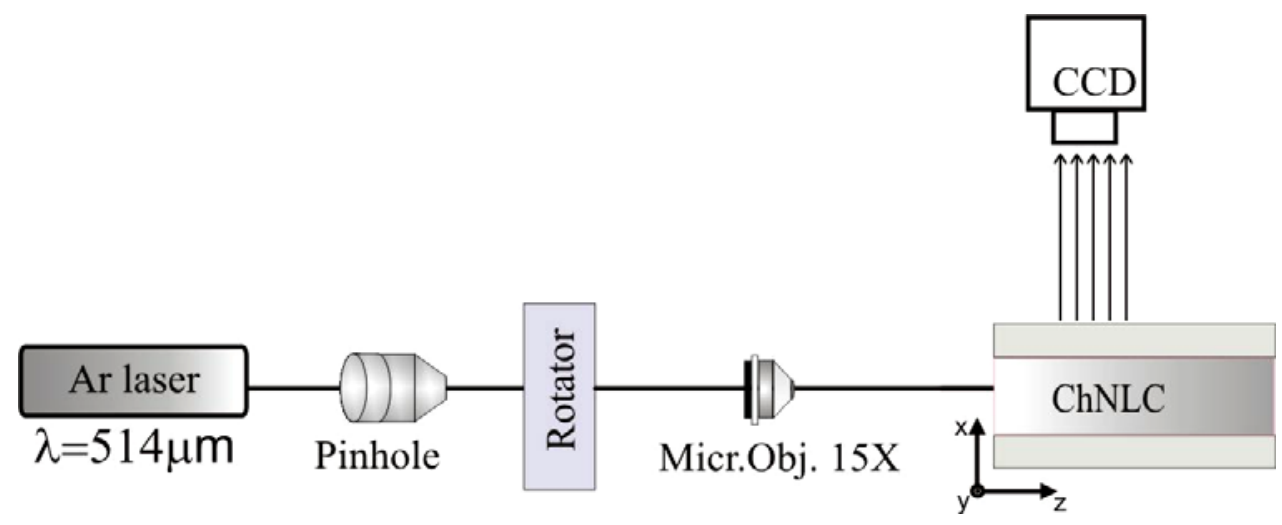

Fig. 2. Schematic drawing of the experimental setup.

Experimental results are depicted in the Fig. 3. In the linear case (signed as $\mathrm{P} \sim 0 \mathrm{~mW}$ ), the diffractive broadening beam propagates in $y z$ plane parallel to the $z$ axis. Increasing the light power leads to the reorientational nonlinear effect and finally causes self-focusing of the beam. For the optical power $\mathrm{P} \sim 35 \mathrm{~mW}$ a beam with nearly unvarying field profile is achieved (see Fig.3c). The solitary beam has a transverse intensity distribution which is unchaned with a propagation distance about $2 \mathrm{~mm}$ (which is over 50 times of the Rayleigh length). Moreover it is shown that only the beam with TE polarization can cause the nonlinear effects: changing for the TM polarization causes beam diffraction (bottom in Fig 3a).

The second experimental setup included two light sources, the Argon pump and the HeliumNeon probe beam, is presented in Fig.4. Trough a pinhole two co-propagating and copolarized beams were coupled into the NLC cell using $15 x$ microscope objective. The cell was placed on the $x-y-z$ stage. The light propagation in the $(y-z)$ plane was analyzed by 
collecting the scattered light from the liquid crystal above the cell. To this extent, we used an optical microscope and a CCD camera, inserting a red filter to let only the He-Ne light through, whenever required.

a)
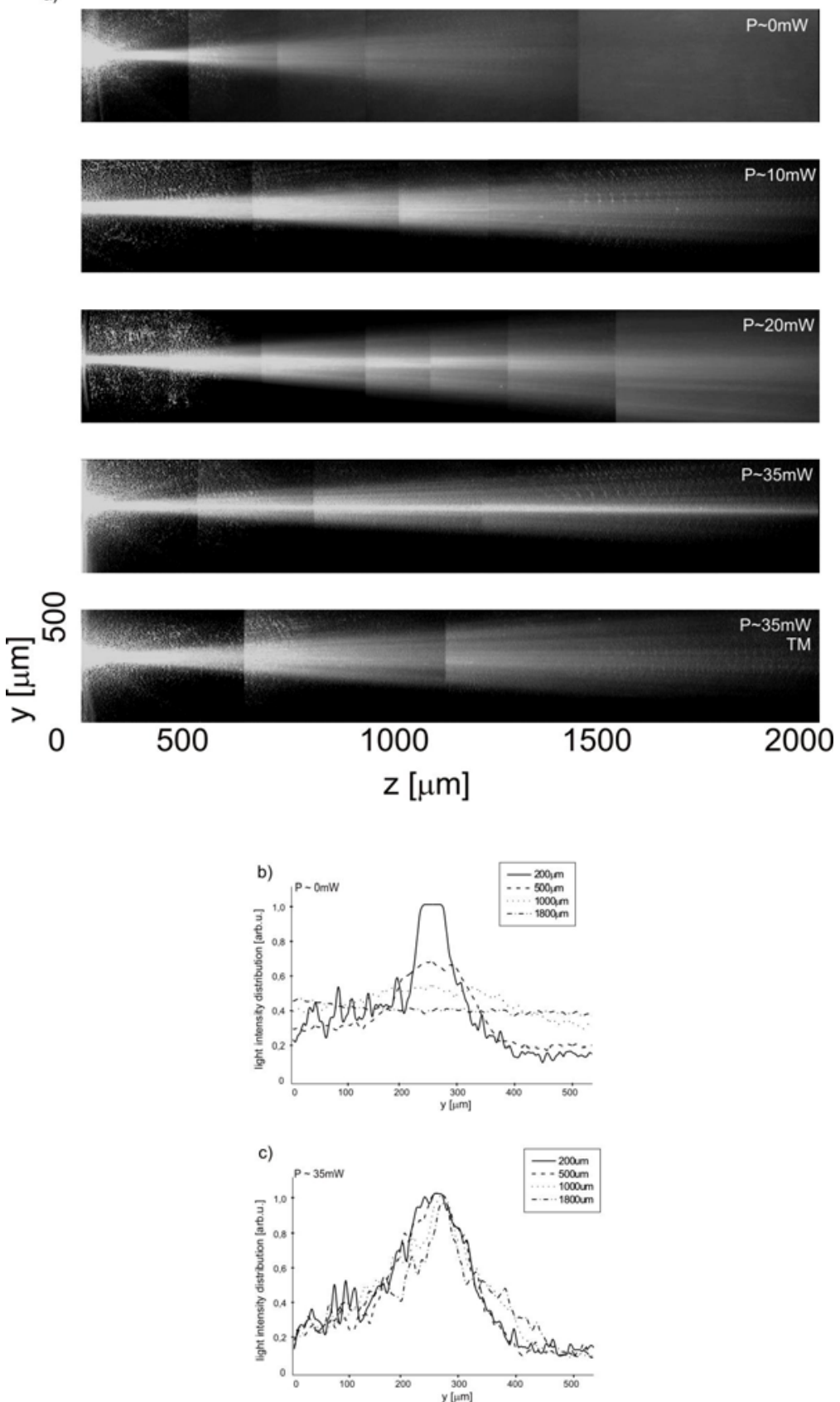

Fig. 3 Experimental results of creation spatial solitons in ChNLCs layer; a) light beam propagation for different inputs of light power (marked on photos) and centrally launching light beam, the last picture was taken for the TM polarization, b)-c) light intensity profiles for low and high power respectively, and for different values of propagation distance, corresponding to photos from (a). 


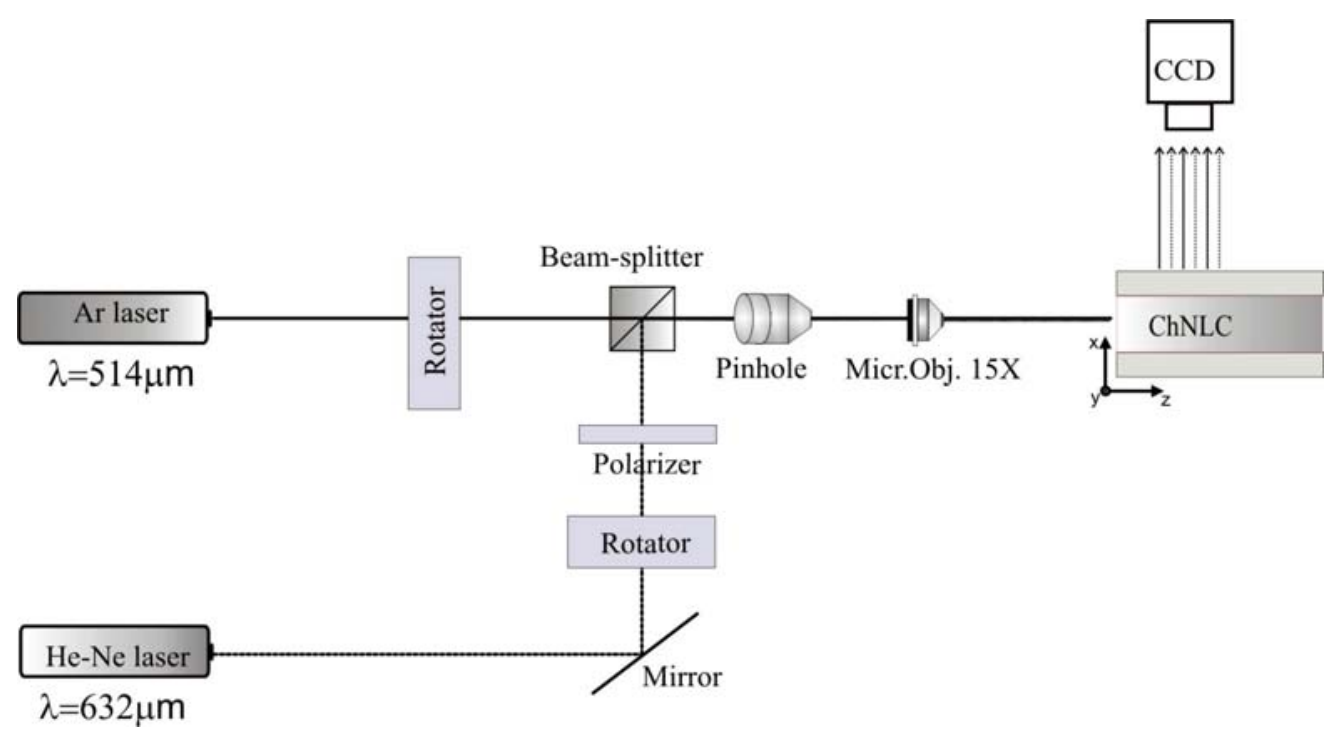

Fig. 4 Schematic drawing of the experimental layout for two-beam measurements.
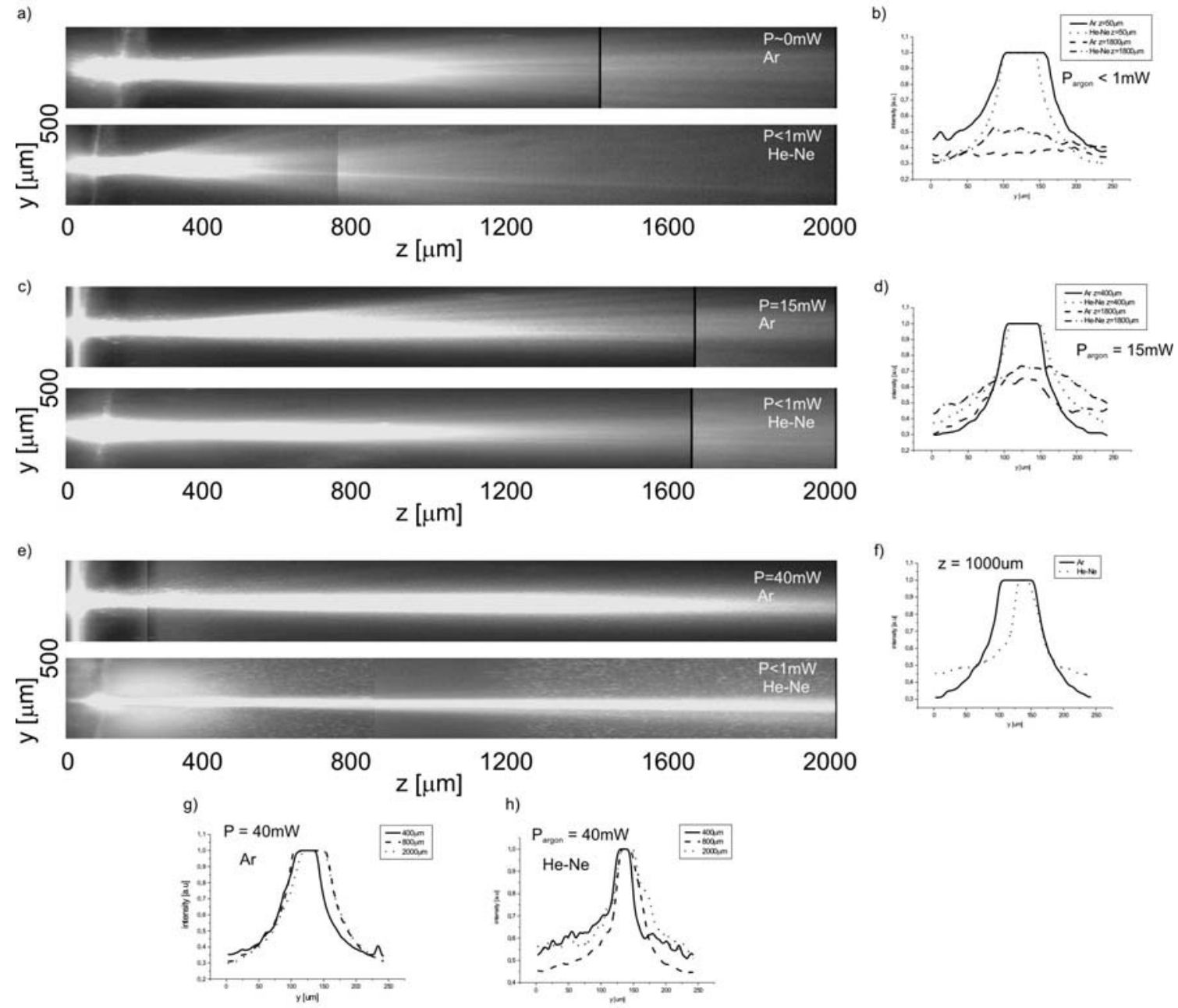

Fig. 5 Propagation of the He-Ne low-power probe beam in the waveguide induced by the Ar beam and corresponding normalized intensity profiles at different propagation distances in the linear diffraction case $(\mathrm{a}, \mathrm{b})$, for the Ar beam power $\mathrm{P}=15 \mathrm{~mW}(\mathrm{c}, \mathrm{d})$ and for $\mathrm{P}=40 \mathrm{~mW}(\mathrm{e}-\mathrm{h})$. 
The spatial soliton formation in the liquid crystal cell introduces changes in the refractive index distribution and leads to the formation an optical waveguide. This was verified by injecting the second co-polarized low-power He-Ne probe beam. When the pump beam diffracts, the probe beam also diffracts (Fig. 5a and corresponding intensity cross-section in the Fig. 5b). Widths of these two co-propagating beams are similar, but they differ due to the different wavelengths. When the TE-polarized beam from argon laser forms a spatial soliton for higher light power, the co-propagating $\mathrm{He}-\mathrm{Ne}$ probe beam is also confined (Fig. 5e). Moreover, the probe beam follows the direction of the soliton formed by the Ar light beam exactly. Increasing the power of the pump causes the lensing effect that focuses the beam, and the probe becomes increasingly confined too (Fig. 5c). When the light power of the pump is high enough to create spatial soliton, the width of the probe beam is also almost constant over the propagation distance (Fig $5 \mathrm{~h}$ ).

Concluding, in recent years it has been proved that nematic liquid crystals can suport spatial solitons at distances of few milimiters for light powers that are a few tenths of a miliwatts. In this paper we have reported the existence of spatial optical solitons in chiral nematic liquid crystals for tenths of $\mathrm{mW}$ input powers over a few millimeters propagation distance. Furthermore we have also presented an optically induced waveguide, and the possibility for trapping and confining the low power probe beam by the pump beam at a different wavelength. The large variety of proposed configurations and types of solitons allow to built different elements with properties necessary for a given application.

\section{References:}

1. Simoni, F., Nonlinear Optical Properties of Liquid Crystals, World Scientific Publ.: London (1997).

2. Karpierz, M. A., Soliton Driven Photonics, Boardman, A. D., Sukhorukov, A.P., (Eds.), p. 41, Kluwer Academic Publishers: Dordrecht (2001).

3. Assanto, G., Peccianti, M., Conti, C., Opt. Photon. News 14, 44, (2003).

4. Karpierz, M. A., Sierakowski, M., Świłło, M., Woliński, T. R., Mol. Cryst. Liq. Cryst. 320, 157, (1998).

5. Karpierz, M. A., Phys. Rev. E 66, 036603, (2002).

6. Peccianti, M., De Rossi, A., Assanto, G., De Luca, A., Umeton, C., Khoo, I. C., Appl. Phys. Lett. 77, 7, (2000).

7. Peccianti, M., Assanto, G., Opt. Lett. 26, 1690, (2001).

8. Peccianti, M., Assanto, G., Phys. Rev. E 65, 035603, (2002).

9. Warenghem, M., Henniot, J. F., Abbate, G., Mol. Cryst. Liq. Cryst. 320, 207, (1998).

10. Derrien, F., Henninot, J. F., Warenghem, M., Abbate, G., J. Opt. A: Pure Appl. Opt. 2, $332,(2000)$.

11. Fratalocchi, A., Assanto, G., Brzdakiewicz, K.A., Karpierz, M.A., Opt. Lett. 29, 1530, (2004).

12. Fratalocchi, A., Assanto, G., Brzdąkiewicz, K.A., Karpierz, M.A., Opt. Express 13, 1808, (2005).

13. Karpierz, M. A., Acta Phys. Polonica A, 99, 161, (2001). 
14. Karpierz, M. A., Sierakowski, M., Wolinski, T. R., Mol. Cryst. Liq. Cryst. 375, 313, (2002).

15. Karpierz, M. A., Brzdąkiewicz, K. A., Nguyen, Q. V., Acta Phys. Polonica A, 103, 169, (2003).

16. Jaworowicz, K., Brzdakiewicz, K. A., Karpierz, M. A., Sierakowski, M., Mol. Cryst. Liq. Cryst. 453, 301, (2006).

17. Ong, H. L., Phys. Rev. A 37, 3520, (1988).

18. Hong, Q., Wu, T.X., Wu, S-T., Liq. Cryst. 30, 367, (2003). 